\title{
Infertility in a Large Royal Air Force General Practice
}

\author{
Squadron Leader T E Martin \\ BSc, MB, BS, DRCOG, RAF
}

British Medical Centre, Ramstein Air Base, BFPO 109

SUMMARY: The presentation and management of infertility was studied in a group of 56 couples from the catchment area of a large Royal Air Force general practice. They represented an overall prevalence of 36 per 1000 couples in the population. Thirty seven couples $(66 \%)$ were trying for their first pregnancy and $19(34 \%)$ has experienced a previous conception. Disorders of ovulation occurred in 29 women $(52 \%)$ whereas disorders of sperm production or function occurred in 21 men (38\%). Other causes were less common. Twelve couples (21\%) had more than one diagnosed cause for their infertility, but 21 (37\%) withdrew voluntarily or were posted back to the UK during the study period. Only 10 couples $(\mathbf{1 8 \%})$ were referred to hospital for specialist investigations or treatment. A logical scheme for the GP management of infertile couples was used. This regime may have been partially or wholly responsible for the 14 pregnancies $(25 \%)$ reported.

\section{Introduction}

It is well known that the inability to conceive may be a cause of intense misery and anxiety for the childless couple. Anecdotal evidence suggested that infertility was a sizeable problem in our practice. The decision to establish a GP controlled fertility "clinic" was aided by the fact that our nearest British hospital is 50 miles to the north. One doctor took the responsibility of supervising and coordinating all the investigations of those couples who attended with problems of conception over an 18 month period. As far as the author is aware, this GP controlled clinic is a new concept.

This study was therefore designed to examine infertility in a busy Royal Air Force (RAF) general practice overseas. There were three main objectives:

1. To investigate the prevalence of infertility over an 18 month period at RAF Gütersloh.

2. To identify causative factors in the infertility of the study population.

3. To compare the results of the above with a stable UK based population.

A parallel study by the same author ${ }^{1}$ has established guidelines for the GP initiated investigation and management of these patients. It is summarised in Figures 1 and 2.

\section{Patients and Methods}

The Station Medical Centre (SMC) at RAF Gütersloh serves a population of around 7,000 military and civilian personnel who live within a 150 square mile area of Westphalia in the Federal Republic of Germany. Most of the servicemen work at the large Royal Air Force Station which is situated just outside the town of Gütersloh. The Station itself is the home of the Harrier and Helicopter forces in RAF Germany, and is subject to frequent and regular deployments which often take servicemen away from their families.

In addition to the servicemen and women of the Royal Air Force and their dependants, the practice caters for UK based civilian employees of the Civil Service and for dependants of serving Army personnel from the nearby Gütersloh garrison.

The calculation of population statistics is somewhat easier than it would be in a civilian general practice. Divorced and widowed women do not remain registered as dependants of servicemen, and therefore the number of couples in this population is, to all intents and purposes, the same as the number of married females registered in the practice. Therefore the following statistics were collected:

a. The total number of "families" registered - an indirect measure of the total number of married female dependants.

b. The total number of married servicewomen.

c. The total number of married professional women who are working at RAF Gütersloh in their own right (ie they are UK based civilians who are not dependants of servicemen).

d. The total number of families with children (taking into account those at boarding school etc).

e. The total number of primigravid antenatal patients.

f. The total number of childless families, calculated by: $(a+b+c)-(d+e)$.

g. The total number of couples under investigation and/or treatment of infertility. Only couples who had failed to achieve a pregnancy after at least 12 months frequent unprotected intercourse were entered into the study group.

The study period was from 25 September 1986 to 25 March 1988. The above totals were calculated after six and twelve months and at the end of the study period.

Unlike many other studies, the prevalence rate is used in preference to the incidence, since prevalence is paricularly useful for describing how much disease (in this case infertility) is present in the community. Some of the couples who presented at RAF Gütersloh had been previously managed by an earlier GP or hospital clinic; these would not have been reflected in figures of incidence. Since percentage figures are also often 
quoted, the percentage of couples attending for the management of infertility has also been calculated taking the total number of married women between the ages of 18 and 45 (46th birthday) as unity (100\%).

The following details were recorded for each couple.

a. Date of presentation.

b. Number of years trying to conceive (at presentation).

c. Primary or secondary infertility.

d. Infertility factors, when known.

e. Present status or stage of management.

Case histories were summarised and tabulated either:

a. On achieving a pregnancy.

b. On referral to a specialist infertility clinic.

c. On posting away from RAF Gütersloh.

d. On voluntary withdrawal from the program.

e. Or at the end of the study period.

The scheme for investigation and treatment of these couples evolved throughout the study period and a logical plan of management was established (Figs 1 and 2). Management was tailored to each individual couple, especially when investigations had already been started by a previous GP or specialist. Each couple was fully informed of what to expect and what would be required from them during the course of the investigations. They all received an overall plan to which they could adhere, but of course, the fine detail of each plan was changed in the light of results as they became known. "Patient advice literature" was often used to reinforce information given at consultations and compliance in both investigations and treatments was generally good.

A portfolio of clinical notes ${ }^{1}$ was designed specifically for the GP management of these couples. The key investigations around which all others were planned were mid-luteal phase progesterone (MLPP), semenalysis and post coital testing of cervical mucus (PCT). It is well known that temperature charting is an imprecise means of predicting ovulation ${ }^{2}$ and in this study the basal temperature chart was used only in conjunction with the charting of changes in the cervix and cervical mucus. Detection of urinary luteinizing hormone was not used.

Treatments at GP level can be divided into two main groups, those directly related to a known infertility factor and those which treat other conditions (medical, surgical, psychological or social) which may be implicated as a possible cause of the infertility. The following list illustrates the extent of treatments offered to couples at RAF Gütersloh.

Treatment of infertility factors:

1. Stimulation of ovulation.

2. Oestrogenisation of hostile cervical mucus.

3. Antibiotic therapy for pelvic inflammatory disease and cervicitis.

4. Suppression of hyperprolactinaemia.

5. Stimulation of spermatogenesis.

6. Suppression of anti-sperm antibodies.
7. Antibiotic therapy for prostatitis and other male genital infections.

8. Artificial insemination by husband (AIH).

Treatment of infertility implicated factors:

1. Insulin and/or diet for diabetes mellitus.

2. Diet for obesity.

3. Thyroid replacement therapy for hypothyroidism.

4. Anti-smoking advice/therapy.

5. Counselling for anxiety, stress and othe psychological or social problems.

Treatments were usually GP initiated, but occasionally a continuation of therapy started in a previous hospital clinic. Details of individual therapies are well? described elsewhere ${ }^{3,4}$.

Three distinct groups of patients were referred for $\stackrel{\omega}{\circ}$ specialist investigations and advice:

1. Those with unexplained infertility after completion of all GP instigated investigations.

2. Those who had a diagnosis which necessitated $\vec{\omega}$ further hospital investigation and/or management.

3. Those who requested referral.

After some couples had been investigated and/or o referred, there remained a small proportion with $\triangle$ unexplained infertility and still others who had sufferf treatment failure or who had an "untreatable" cause far their infertility. These couples required carefal ${ }^{\Phi}$ counselling, sympathetic advice and help in coping wi $\overrightarrow{0}$ their anguish.

\section{Results}

For the reasons outlined above (see methods), the number of couples was assumed to be equivalent to the number of married women within the age range of 18 to $\mathbb{Q}$ 45 years. The number of couples with proven fertility $\overrightarrow{\vec{F}}$ was taken as the total number of women with children plus the number of primigravid women in the study population. These totals were calculated after 6,12 and 18 months of the study period. The total population at $\stackrel{-}{-}$ RAF Gütersloh was 6609 and 6627 in March and September 1987 respectively, and 6712 at the end of the study period. Table 1 gives the population statistics for the study period.

The number of married women (and therefore also the number of couples) with unproven fertility and the? number of couples attending the fertility clinic were then 3 . compared, and the percentages of both were calculated with respect to the total number of couples in the study 3 population. The results are given in Table 2.

Among the 56 couples studies, the average duration of time trying to achieve a pregnancy (at the time of presentation) was 2.8 years (range 1-10 years). Fourteen couples $(25 \%)$ achieved a pregnancy during $N$ the study period. One (a twin pregnancy) resulted from $N$ IVF. Of the others (all singleton pregnancies), seven occurred whilst treatment by induction of ovulation was 
Temperature Chart and examination of cervical mucus

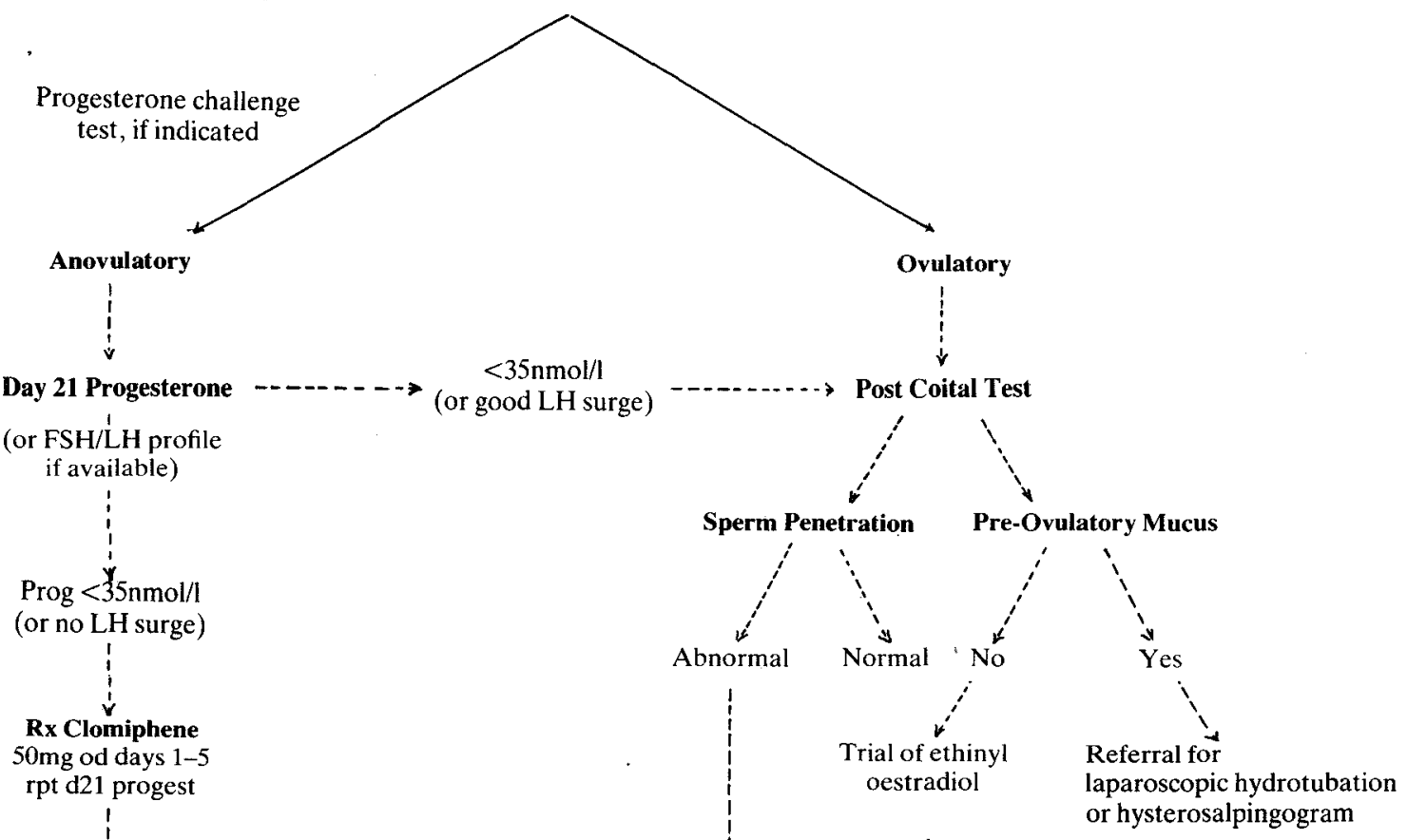

Still $<35 \mathrm{nmol} / 1$ (or good LH surge)

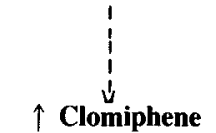

$100 \mathrm{mg}$ od days $1-5$ rpt d21 progest<smiles>[Li][As](I)I</smiles>

(or good LH surge)

If abnormal, further investigations and treatment as required.

Fig. 1. Investigation and Management Scheme for Female Infertility.

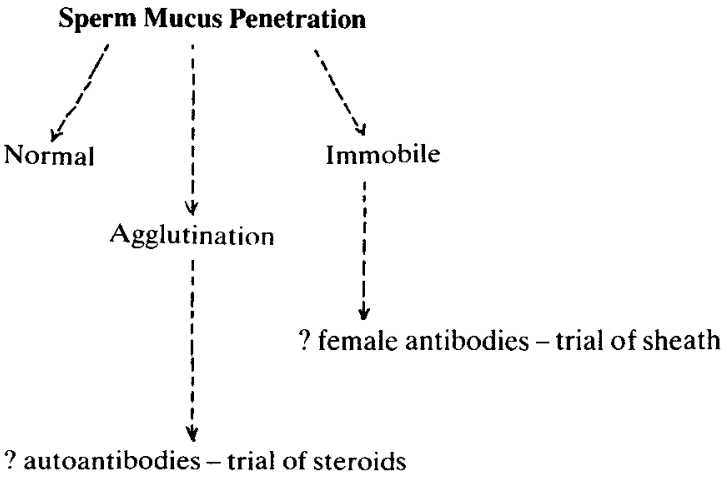




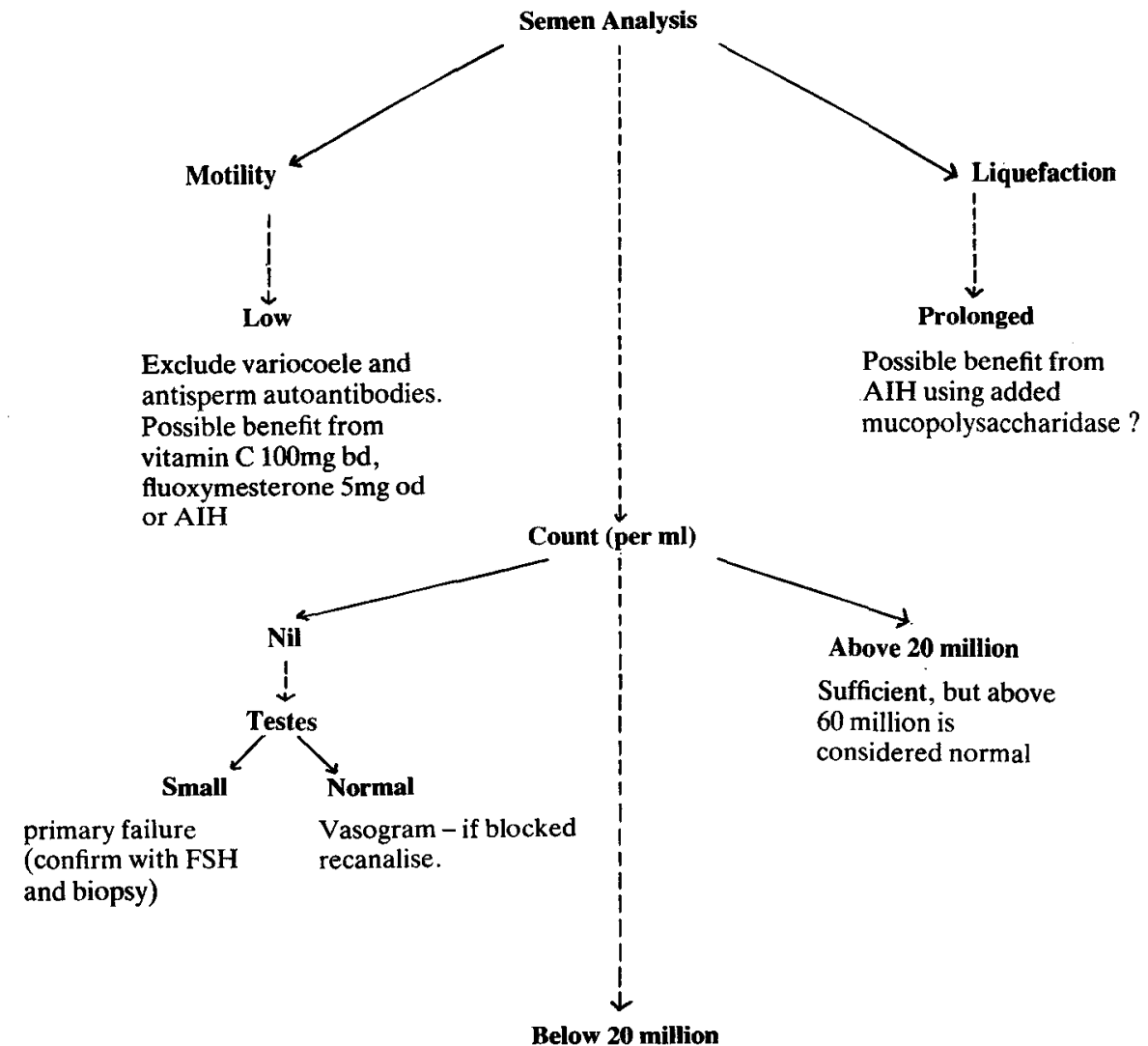

If FSH low - Rx tamoxifen 10mg od.

If testosterone low - Rx mesterolone $100 \mathrm{mg}$ od.

If prolactin high - exclude pituitary tumour.

If varicocele present - repair.

Otherwise may require AIH, AID or IVF.

\section{Notes:}

a. Many of the treatments described above are known to improve count, motility or liquefaction time, but few seem to improve the overall chances of conception.

b. MSU and/or semen, MC\&S may be required to exclude infection.

c. If $>60$ milion, $>80 \%$ motile, $>20 \%$ abnormal and liquefaction time is normal then no further investigation of the husband is required.

Fig. 2. Investigation and Management of Male Infertility.

in progress. One woman was also receiving bromocriptine treatment for hyperprolactinaemia and another was being treated for diabetes mellitus. The only medical intervention received by the remaining six couples was the teaching of the principles of ovulatory prediction and the appropriate timing of intercourse. Their pregnancies occurred whilst investigations were still in progress. The average time that these latter $\underset{D}{D}$ couples took to conceive was 5.5 months from first attendance (range 1-9 months) and 21 months from first $N$ trying (range 13-30 months).

The average age of the husbands was 27.6 years (range 21-47 years), whereas that of their wives was 27.7 years $\mathrm{c}$ (range $20-45$ years). Thirty seven of the couples $(66.1 \%)$ ? 
Table 1

\begin{tabular}{lrrr}
\hline Sub-Group & 25 Mar 87 & 25 Sep 87 & 25 Mar 88 \\
\hline Married dependant females & 1519 & 1550 & 1537 \\
$\begin{array}{l}\text { Married servicewomen } \\
\text { Married civilian }\end{array}$ & 35 & 37 & 28 \\
non-dependants & 2 & 2 & 2 \\
\hline Total Married Females & $\mathbf{1 5 5 6}$ & $\mathbf{1 5 8 9}$ & $\mathbf{1 5 6 7}$ \\
(= Total Married Couples) & & & \\
\hline $\begin{array}{l}\text { Women with children } \\
\text { Primigravid women }\end{array}$ & 1088 & 1017 & 1045 \\
Total Women (Couples) & 49 & 40 & 34 \\
with Proven Fertility & 1137 & $\mathbf{1 0 5 7}$ & $\mathbf{1 0 7 9}$ \\
\hline
\end{tabular}

Table 2

\begin{tabular}{lccc}
\hline Sub-Group & 25 Mar 87 & 25 Sep 87 & 25 Mar 88 \\
\hline $\begin{array}{l}\text { Total Couples } \\
\text { Total couples with unproven }\end{array}$ & 1519 & 1550 & 1537 \\
fertility & 419 & 532 & 488 \\
$\begin{array}{l}\text { Percentage of couples with } \\
\text { unproven fertility }\end{array}$ & $27 \%$ & $33 \%$ & $31 \%$ \\
$\begin{array}{l}\text { Cumulative total couples } \\
\text { attending fertility clinic }\end{array}$ & 18 & 39 & 56 \\
$\begin{array}{l}\text { Percentage of couples } \\
\text { attending clinic }\end{array}$ & $1.2 \%$ & $2.5 \%$ & $3.6 \%$ \\
\begin{tabular}{l} 
Prevalence of infertility \\
\hline
\end{tabular} & 0.012 & 0.025 & 0.036 \\
\hline
\end{tabular}

were trying to achieve their first pregnancy. The average age of these nulligravid women was 27.0 years (range $20-45$ years). The remaining 19 couples $(33.9 \%)$ had already achieved a pregnancy, and were regarded as suffering from secondary infertility, whatever the outcome of the previous pregnancy (or pregnancies). The average age of these women was 28.9 years (range $22-45$ years).

Table 3 gives the distribution of causes of infertility diagnosed in the study population (when known). Twelve couples $(21.4 \%)$ had more than once cause for their infertility and investigations in 31 couples $(55.4 \%)$ were incomplete at the end of the study period. Of these, 7 couples $(12.5 \%)$ were posted back to the UK before investigations were completed and 14 couples (25\%) voluntarily withdrew from the program. Ten couples $(17.9 \%)$ were still being investigated at the end of the study period.

Ten couples $(17.9 \%)$ were referred for specialist investigations or hospital management, and two (3.6\%) were referred to an adoption agency. Two couples $(3.6 \%)$ were accepted on to IVF/GIFT programs in the UK.

Disorders of ovulation included oligomenorrhoea $(14 \%)$, PCOS $(11 \%)$ and luteal insufficiency diagnosed by MLPP persistently lower than $35 \mathrm{nmol} / 1(27 \%)$. None of the women had amenorrhoea. Of the sperm disorders, there were two cases of azoospermia (Klinefelter's syndrome and primary failure of spermatogenesis), oligozoospermia was found in $29 \%$ and normal semenalysis with negative PCT occurred in $5 \%$. One of these patients had prolonged liquefaction time and another was found to have antisperm autoantibodies. Miscellaneous causes included hyperprolactinaemia, hypothyroidism and diabetes mellitus. No cases of coital failure, psychosexual disorder or endometriosis were diagnosed in the study population.

\section{Discussion}

An attempt has been made to describe infertility in a defined population. Data was collected on all couples attending the medical centre with inability to achieve conception during an 18 month period from 25 September 1986 until 25 March 1988.

A recent study by Hull $^{5}$ et al demonstrated an annual incidence of 1.2 couples per 1000 of the population, and revealed that $17 \%$ of all couples seek medical advice for infertility at some time in their lives. At the beginning of the study the RAF Gütersloh figure was only $1.2 \%$ despite $33 \%$ of married couples having unproven fertility. By the end of the study period this figure had reached $3.6 \%$. There is no direct comparison between point prevalence and annual or lifetime incidence, but prevalence is obviously a better indicator of how much infertility is in the community. The true number of infertile or subfertile couples is probably even higher than $3.6 \%$, so why the difference? There are a number of dissimilarities between service and civilian populations which must be considered.

The first and probably most important difference between comparably sized National Health Service (NHS) and service populations is that the latter is transient and mobile. Job and house moves occur frequently, occasionally as often as 6 monthly, but more commonly at intervals of 2 to 4 years. Husbands often work night shifts and may be subject to frequent deployments away from home. Some of these detachments last up to 4 months, a fact which must be taken into account when deciding if true infertility really does exist.

\section{Table 3}

\begin{tabular}{lcc}
\hline Cause of Infertility & $\begin{array}{c}\text { No Couples } \\
\text { with Each Case }\end{array}$ & $\begin{array}{c}\text { \% of Couples } \\
(\mathbf{n = 5 6 )}\end{array}$ \\
\hline $\begin{array}{l}\text { Disorders of ovulation } \\
\text { Disorders of sperm }\end{array}$ & 29 & 51.8 \\
production or function & 21 & 37.5 \\
Cervical mucus & 1 & \\
dysfunction & 3 & 1.8 \\
Tubal damage & 5 & 5.4 \\
Miscellaneous & $\mathbf{5 9}$ & 8.9 \\
\hline Total No Causes Diagnosed & \\
\hline
\end{tabular}


Secondly, servicemen (if not their families) are generally a healthy population, since all must have passed a recruiting medical examination before enlistment. This should exclude all chronic medical and debilitating conditions.

Lastly, most of the study population are enlisted for a career. Unemployment does not exist, and although RAF families may not be affluent, they are certainly not poor. Each family has at least one wage earner and every married couple will be housed in a married quarter or private hiring. Social circumstances are generally favourable and social problems form only a small part of the RAF GP's workload.

The following reasons for the apparently low prevalence of infertility in the study population are suggested:

a. Much infertility is masked by career-minded longterm contraception users.

b. Many couples try to make the most of their overseas posting and prefer to delay starting their family until return to the UK, thus further masking some cases of infertility.

c. Contraceptive compliance is good in a generally well-educated population, and contraceptive failures in infertile couples will not be obvious.

d. Many of these who do not conceive will prefer to simply "carry on" trying until posted back to the UK, by which time some will have successfully achieved a pregnancy and others will indeed require investigation.

e. There is a higher proportion of younger couples in a service population. One would not expect these couples to be the ones who are most affected. Evidence from this and another study ${ }^{5}$ reveals that the average age for presentation with infertility is around 28 years and many affected couples are in their thirties or forties.

f. Service couples overseas are not able to choose their own GP, although several might be available in the military medical centre. The relatively short tour length means that few know their GP well and even fewer have had time to build a rapport with him. Infertility is an emotional and often embarrassing condition which necessitates trust and understanding between doctor and patients. Some patients will, no doubt, be reluctant to approach a GP whom they hardly know for a condition which, by necessity, involves personal and intimate questioning and investigation of both partners.

The specific problem of being a mobile and transient population may adversely affect the medical care given to our service patients. Many of these couples have been seen in several other medical establishments before reaching our unit. Valuable time is often lost as simple (but time consuming) investigations are needlessly repeated. To overcome this duplication and the ubiquitous problem of lost or delayed notes, each of the couples investigated for infertility at RAF Gütersloh is given a complete portfolio of their own case history when they leave our care or are referred to a hospital specialist. All results are recorded, since negative findings are as important as positive ones.

Like Hull's work ${ }^{5}$, this study examines both primary and secondary infertility in the population. The distributions are similar in both studies, despite Hull's population being from a West Country hospital clinic. Women who had never achieved a pregnancy were, on average, only two years younger than their contemporaries who had previously conceived. A previous pregnancy was, however, rare in women whose husbands had a clearly demonstrable disorder of sperm production or function, thus suggesting that the disorder is persistent.

Other findings described by $\mathrm{Hull}^{5}$ have also been confirmed. Six women in the study population $(11 \%)$ had polycystic ovary disease (PCOS). This is a surprisingly high number and supports the statement that PCOS is a "major cause of infertility". In contrast, the study population contained no couples with coital failure and no women with endometriosis. This, in turn, confirms Hull's findings that these conditions are "infrequently the cause of infertility".

One striking dissimilarity between the two studies io the surprisingly low number of cases of unexplained infertility in the RAF population (15\%) compared witk the NHS population $(28 \%)$ ). The most likely explanation for this finding is that the study group was small and the number of couples with incomplete investigations was large $(55 \%)$. Those with unexplained infertility were referred for specialist management. They had normat and frequent sexual intercourse, normal semenalysis; MLPP above $35 \mathrm{nmol} / \mathrm{l}$, penetrative sperm on PCT and no abnormality detected on laparoscopic hydrotubation. Haxon et $\mathrm{al}^{6}$ suggest that patients with unexplained infertility may have been inadequately investigated in the first instance. In a study of 95 couples with 3 or more years of unexplained infertility, reinvestigation led to definite diagnoses in over $63 \%$. This view has been supported by Winston and Margara ${ }^{7}$.

Thoroughness during initial investigation was shown to produce good results in this study. Fourteen pregnancies achieved during the study period is $25 \%$ of the cumulative total of all couples who attended the fertility clinic. Taking into account those who were posted or otherwise withdrew from the study program, the success rate is an impressive $56 \%$. Half of these pregnancies resulted from positive medical intervention. This compares favourably with the "less than $18 \%$ " who may conceive as a "result of medical intervention excluding IVF and GIFT" quoted by Lilford" .

Inevitably, our referral centre also benefited by virtue of a lightened workload. Before this study was undertaken it was the policy at our practice to refer all couples with problems achieving a pregnancy to the gynaecology clinic. During the study period only 10 政 
couples $(18 \%)$ were referred, and two of these were referred directly to an IVF centre in the UK.

The most commonly defined cause of infertility was disordered ovulation (52\%), unlike Hull's population in which defective sperm function predominated ${ }^{5}$. Nevertheless this was the second commonest group of causes at RAF Gütersloh with $38 \%$ of husbands affected. Forty eight per cent of women whose husbands had a disorder of sperm production or function had a low MLPP (<35nmol/1), confirming that multiple causation of infertility is not uncommon?

Of the pregnancies successfully achieved, seven occurred during treatment with clomiphene. Due to the short duration of this study, a two year success rate could not be calculated. However, of 29 women with ovulation disorder (confirmed by MLPP assay ${ }^{10}$ ), eight remained untreated (because of voluntary withdrawal or posting, etc) and none of the remainder had taken clomiphene for more than 12 months (at the close of the study period). The apparent success rate for clomiphene is therefore likely to be better than $33 \%$. Hull ${ }^{5}$ showed that the cumulative conception rate in couples with oligomenorrhoea as the only cause of infertility was almost $74 \%$ over 12 clomiphene treatment cycles.

In this study nine of the 21 women treated with clomiphene had a second cause for their infertility, or their partner had a proven defect of spermatogenesis. Five of the six patients with confirmed PCOS received clomiphene. Three of these women achieved a pregnancy within 6 months of starting treatment. Despite the low numbers, this appears to contradict Bromham's statement that "even among (PCOS) patients with an ovulatory response to clomiphene, conception rates remain low"1!.

GP initiated treatments for oligozoospermia, poor liquefaction and antisperm antibodies did not result in a successful pregnancy. Hull ${ }^{5}$ stated that the overall chance of natural conception for these couples is about $20 \%$ over the course of two years. Six women in this study achieved a pregnancy whilst investigations were still in progress. Of these, only one husband had oligozoospermia. Another couple, in which the husband had profound oligozoospermia, achieved a successful pregnancy after a single treatment cycle of IVF.

Four women with negative PCT in the presence of normal ovulation underwent a trial of artificial insemination using spun washed ejaculates of husbands' sperm introduced into the uterus twice each cycle in the pre-ovulation phase (as determined by timing and mucus examination). The average duration of treatment was 3 cycles (range $2-5$ cycles). No pregnancies were achieved. Glazener et $\mathrm{al}^{12}$ have reported that high AIH is of no benefit in the treatment of male infertility nor in cases of negative PCT when semenalysis was normal. Donor insemination was not available to the patients in this study.

The six women who conceived during investigations had received no treatment except for instruction on the detection of the most fertile phase of their cycle and timing of intercourse. It is quite likely that some, if not all, of these women would have conceived regardless of their instruction. The average time taken for these women to conceive was 5.5 months from attendance and 21 months from first trying. Tietze ${ }^{13}$ estimates that $10 \%$ of all fertile couples take longer than one year to achieve a pregnancy even in the presence of frequent unprotected intercourse.

There is no doubt about the significant increase in workload that occurred once it became known that our practice had a special interest in fertility problems. Before the establishment of the Gütersloh clinic, all infertile patients were referred for specialist advice after the first or second consultation. During the first six months of the study 18 couples sought advice for infertility and most were subsequently managed at GP level and without recourse to hospital referral. Even taking into account those couples who withdrew, were posted or achieved a pregnancy, the numbers attending the clinic at the close of the study were double what they were at the end of the first 6 months.

Despite the amount of time required to manage these patients, there can be no doubt that the GP has a major role to play in the initial evaluation of the infertile couple and, if he is experienced, even in the subsequent medical management, whether it be GP or hospital initiated. There are many reasons why the GP may be the doctor best suited for this job:

a. He may already know the couple and a rapport may already exist.

b. The couples can be seen more frequently than in a hospital outpatient system and more easily at 6 specific times in the wife's menstrual cycle.

c. The GP should be able to devote more time than the hospital clinician.

d. There is no requirement for long-distance travel to hospitals for simple tests and advice.

e. The couple have easier and quicker access to their results (which they may discuss at their ease with a GP whom they may know well).

f. Continuity of care. One doctor sees the couple throughout.

One might ask how relevant the results are, and if this study population is representative of the overall UK population. Inaccuracies are inevitable in such a study, and most occur in the compilation of the population statistics. For the purpose of this study, a number of assumptions were made:

a. All members of the population at RAF Gütersloh are registered at the station medical centre.

b. The number of couples in the study population is equivalent to the number of married women. There were no permanently separated, divorced nor widowed women in this group. No single women (daughters of servicemen and professional civilian non-dependants) were included, since none 
attended for investigation of infertility during the study period.

c. The "fertile years" were taken as being between the 18 th and 46th birthdays. The only basis for using these limiting age brackets was anecdotal, in that during the 12 month study period all the women attending for ante natal care were aged 18 to 45 .

d. The population statistics only identify childless couples as potential sufferers of infertility. All couples with children are said to have "proven fertility". The study takes no account of those couples who already have children but are unable to produce a subsequent pregnancy. If these couples do not identify themselves by seeking medical advice there is no way of accurately estimating the prevalence of secondary infertility in the study population.

e. Married servicewomen are childless, by choice, for career reasons, until proven otherwise. (Servicewomen may be pregnant whilst still on RAF duties, but are released from the service before the baby is born).

In addition the study fails to take into consideration those who have no children yet who have a history of still-birth, miscarriage or termination of pregnancy, those whose children may have been adopted or otherwise removed from their care and those who are primigravid but not yet booked.

Many investigations (and most treatments) are cycle dependent and, since there may be only 11 or 12 cycles per year, a constraint is placed upon the number of investigations that can reasonably be undertaken. Effectively, this means that most couples who first attended in the latter half of the study period had not completed even the most basic and routine baseline investigations. Thus the high number of couples that remained incompletely investigated $(55 \%)$ at the end of this study suggests that 18 months is far too short a study period, and one might be forgiven for questioning the validity of the results. Nevertheless the information so far derived has been useful in the planning of future services for these patients and goes some way to demonstrating how much can be done by the interested general practitioner.

\section{REFERENCES}

1. MARTINTE. Infertility in general practice - its prevalence and management. Unpublished Syntex Award regional essay winner. 1987.

2. BAUMAN J E. Basal body temperature: unreliable method of ovulation detection. Fertil Steril 1981; 36: 729-33.

3. Phillipp E E and Carruthers G B. Infertility. London: Heinemann, 1981.

4. Hull MGR, Savage P E and Bromham D R. Anovulatory and ovulatory infertility: results with simplified management. Br Med J 1982; 284: 1681-5.

5. Hull M G R, et al. Population study of causes, treatment and outcome of infertility. Br Med J 1985; 291: 1693-7.

6. HAXTON M J, et al. Unexplained infertility - results of secondary investigations in 95 couples. $\mathrm{Br} J$ Obstet Gynaecol 1987. 94: 539-42.

7. WINSTON R and MARGARA R. Effectiveness of treatment for infertility (letter) Br Med J 1987; 295: 608-9.

8. LILFORD R J and DALTONM E. Effectiveness of treatment for infertility. $\mathrm{Br} \mathrm{Med} \mathrm{J} \mathrm{1987;} \mathrm{295:155-6.}$

9. VERE M F and JOYCE D N. Luteal function in patientso seeking AID. Br Med $J$ 1979; 2: 100.

10. WATHEN N C, et al. Interpretation of single progesterone measurement in diagnosis of anovulation and defective luteal phase: observations on analysis of the normal range. Br Med J 1984; 288: 7-9.

11. BROMHAM D R. New infertility treatments. Update 1988 36: 2565-76.

12. GLAZENER CMA, et al. The value of artificial in 0 semination with husband's semen in infertility due to failure of postcoital sperm-mucus penetration-controlled trial of treatment. BrJ Obstet Gynaecol 1987; 94: 774-8.

13. TrETZE C. Statistical contributions to the study of human fertility. Fertil Steril 1956; 7: 88-95.

14. HendRY $W$, et al. The results of intermittent high dose steroid therapy for male infertility due to antisperm antibodies. Fertil Steril 1981; 36: 4-7. 